\title{
A technique for the evaluation of failed fallopian tube ligation with metal clips
}

\author{
N Leonard, W H B Mawhinney, A J Malcolm
}

\begin{abstract}
The evaluation of fallopian tubes after failed tubal ligation can be difficult because conventional histopathological techniques are unable to section the metal clips when in situ. Once the clips have been removed, any evidence of tube patency is lost. This report describes a technique of embedding and sectioning that enables sections to be made while the metal clips are still in situ. This is a modification of a method first described to embed mineralised bone and involves the use of plastic embedding and a diamond saw. Using this technique, a permanent record is made of the tube location and patency.

(7 Clin Pathol 2000;53:400)
\end{abstract}

Keywords: sterilisation failure; fallopian tubes; contraception

The failure rate for laparoscopic sterilisation using metal clips varies between less than $1 \%$ and $4.5 \% .^{12}$ Therefore, the pathologist will occasionally be faced with the problem of evaluating a fallopian tube ligation that has failed and resulted in a pregnancy. The usual procedure is to send both fallopian tubes, often removed at caesarian section, for analysis with the clips still in situ. It is impossible to examine the portions of the tubes within the clips by standard histological methods and, once the clips have been removed, the evidence is largely destroyed.

We describe a technique that enables the clip to be sectioned when still around the tube, thus enabling comment to be made about the exact location and state of the tube lumen. We describe the case of a 31 year old woman sterilised one year previously using Filchie clips. She became pregnant the following year. Both fallopian tubes were removed at caesarian section.

Figure 1 shows the right fallopian tube with a Filchie clip around most of the tissue. Unfortunately, an abnormally small tube is located just outside the clip. A patent lumen can clearly be seen. The use of plastic embedding has clearly shown the cause of the sterilisation failure.

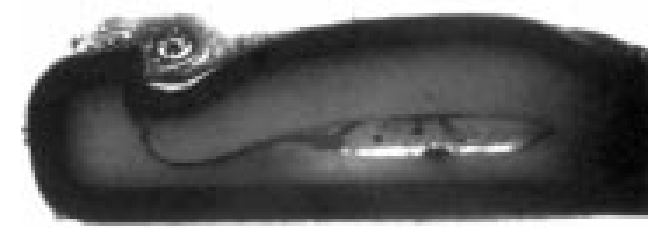

Figure 1 Photograph showing the closed Filchie clip with an abnormally small fallopian tube located outside the clip. A patent lumen is clearly seen.
This technique has been reported previously in the analysis of mineralised bone. ${ }^{3}$ The method involves embedding the specimen in the plastic resin Norpol 340-500, which is a polyester resin incorporating a styrene monomer stabilised by hydroquinone. When an organic peroxide catalyst is added it polymerises the resin by addition reactions between unsaturated $\mathrm{C}=\mathrm{C}$ bonds in the polyester polymer crosslinked with the $\mathrm{C}=\mathrm{C}$ bonds in the styrene. The resulting polymer is inert and forms a hard insoluble plastic that allows most staining reactions commonly used in histology laboratories. The plastic itself does not take up stains and enables sections of suitably sized tissues to be cut down to $2 \mu \mathrm{m}$ in thickness.

\section{Methods}

(1) Fix tissues in $10 \%$ buffered formalin.

(2) Dehydrate in ethanol until test shows all buffer salts are removed. ${ }^{3}$

(3) Infiltrate in three changes of $100 \%$ resin in 24 hours.

(4) Infiltrate for eight hours in agitator in $100 \%$ resin with $1 \%$ Butanox 50 "catalyst" and $1 \%$ hydroquinone in ethanol.

(5) Embed in siliconised glass mould and leave in waterbath heatsink at room temperature overnight (or until polymerised).

(6) Harden in $56^{\circ} \mathrm{C}$ oven for 48 hours.

(7) Remove block from mould and trim off excess resin with a bandsaw and sander.

(8) Cut a $350 \mu \mathrm{m}$ section from the plastic block/tissue using a diamond saw (300 $\mu$ m blade, Struers Accutom saw).

(9) Stick one side of the section to a $2 \times 2 \times 1 / 8$ inch perspex "slide" using cyanoacrylate adhesive and allow to harden (one hour at $37^{\circ} \mathrm{C}$ ).

(10) Polish the exposed face of the section using progressively fine polishing papers on a Struers DAP-7 (grades 120, 320, 1000, and 4000) lapping and polishing machine or equivalent.

(11) Stain the section face in $0.25 \%$ aqueous toluidine blue solution for one hour at $60^{\circ} \mathrm{C}$.

\section{Conclusions}

The use of plastic embedding enables the analysis of the tube with the metal clip in situ. Comment can be made about the exact location of the tube and the state of its lumen.

1 Stovall TG, Ling FW, O'Kelley KR, et al. Gross and histological examination of tubal ligation failures in a residency training program. Obstet Gynecol 1990;76:461-5.

2 Chi IC, Siemens AJ, Champion CB, et al. Pregnancy following minilaparotomy tubal sterilization-an update of an ing minilaparotomy tubal sterilization-an update

3 Mawhinney WHB, Ellis HA. A technique for plastic embedding of mineralised bone. F Clin Pathol 1983;36:1197-9. 